\title{
Dynamic Active Constraints for Hyper-Redundant Flexible Robots
}

\author{
Ka-Wai Kwok ${ }^{1}$, George P. Mylonas ${ }^{1}$, Loi Wah Sun ${ }^{1}$, Mirna Lerotic ${ }^{1}$, \\ James Clark ${ }^{1}$, Thanos Athanasiou ${ }^{2}$, Ara Darzi ${ }^{3}$, and Guang-Zhong Yang ${ }^{1}$ \\ ${ }^{1}$ Royal Society/Wolfson Medical Image Computing Laboratory, \\ Institute of Biomedical Engineering \\ ${ }^{2}$ Department of Bio-Surgery and Surgical Technology \\ ${ }^{3}$ Department of Surgical Oncology and Technology, \\ Imperial College London, London, United Kingdom \\ \{k.kwok07, george.mylonas, 1. sun, mirna. lerotic,j.clark, \\ t.athanasiou,a.darzi,g.z.yang\}@imperial.ac.uk
}

\begin{abstract}
In robot-assisted procedures, the surgeon's ability can be enhanced by navigation guidance through the use of virtual fixtures or active constraints. This paper presents a real-time modeling scheme for dynamic active constraints with fast and simple mesh adaptation under cardiac deformation and changes in anatomic structure. A smooth tubular pathway is constructed which provides assistance for a flexible hyper-redundant robot to circumnavigate the heart with the aim of undertaking bilateral pulmonary vein isolation as part of a modified maze procedure for the treatment of debilitating arrhythmia and atrial fibrillation. In contrast to existing approaches, the method incorporates detailed geometrical constraints with explicit manipulation margins of the forbidden region for an entire articulated surgical instrument, rather than just the end-effector itself. Detailed experimental validation is conducted to demonstrate the speed and accuracy of the instrument navigation with and without the use of the proposed dynamic constraints.
\end{abstract}

\section{Introduction}

Robotic-assisted minimally invasive cardiac surgery has been the major aim of many robotic platforms. The tight confines of the thoracic cavity and mediastinum however, challenge even the most skilled surgeons as limited field-of-view and restricted maneuverability command advanced manual dexterity and hand-eye coordination. These difficulties become even more evident during beating heart surgery, raising critical issues with respect to procedural safety and precision. This is of particular concern during procedures such as minimally invasive bilateral pulmonary vein isolation performed as part of the modified maze approach, to the treatment of chronic and paroxysmal atrial fibrillation when the heart is not only in motion but also beats irregularly. The maze procedure describes the transmural epicardial ablation of a complete circle around the pulmonary veins, an area of the heart known to generate aberrant arrythmogenic electrical activity central to the pathophysiology of atrial fibrillation. Currently, the treatment of atrial fibrillation is through an endovascularly approached endocardial ablation or during open heart surgery with poor response rates [1]. The 
maze procedure is more effective but requires either a median sternotomy or multiple small incisions on both the left and right sides of the chest to ensure that complete encirclement of the vessels has been correctly affirmed [2]. For access via a minimally invasive approach, a catheter is required to pass through two narrow spaces posterior to the heart, tightly bordered by the great vessels. These spaces are roofed by the approximated pericardium. The challenge of ensuring the path for the catheter is accuracy, reflecting upon both the lack of visual field and maneuverability within these spaces. The risk of accidental instrument injury is hampering its uptake despite recognized beneficial patient outcomes.

In robot-assisted procedures, the surgeon's ability can be enhanced by augmented manipulation guidance such as virtual fixtures or active constraints to enhance human sensory feedback and limit aberrant instrument maneuvers [3, 4]. Such concepts have been used to guide the cutting of the tibia and the removal of the blockages within a permitted region in orthopedic knee surgery [5] and endoscopic sinus surgery [6], respectively. They enable the operator to manipulate the tool tip along the desired 3D path without applying excessive force on the contact surface of delicate surroundings. For complex geometries, spatial motion constraints have to be adapted in real-time, making them suitable for surgical interventions. Previous work has so far mainly relied on the preoperative segmented image data or an anatomical model. Moreover, the constraints are assumed to be static throughout the operation. This, however, is not practical especially in cardiac procedures, which involve large respiratory and cardiac induced tissue deformation. The future clinical impact of active constraints or virtual fixtures requires not only flexibility in updating the constraints, but also the ability to react to changes in tissue morphology intra-operatively. Recently, virtual fixtures have addressed dynamic surgical scenes where tissue deformation is present. For example, Ren et al. [7] proposed dynamic 3D virtual fixtures for beating heart ablation procedures. The work complements manipulation guidance with intra-operative sensing data. The dynamic virtual fixture model is defined based on the pre-operative data, then registered to intra-operative images. Most of the existing methods, not excluding [6], are concerned with confining the motion of a single-point end-effector rather than the instrument body, even if it is rigid. Current research into roboticassisted cardiac surgery has been focused on developing more flexible hyperredundant devices to overcome the problem of safe access and navigation within the tight confines of the chest cavity and pericardial space $[8,9]$. Deformation of the heart can have a profound effect on spatial constraints. To overcome these difficulties, dynamic active constraints could permit rapid and safe transthoracic and intrapericardial navigation without injuring the surrounding tissue or organs.

The purpose of this paper is to introduce a real-time modeling scheme for dynamic active constraints with adaptation to cardiac deformation and anatomic structure changes. A smooth and dynamic cylindrical pathway with detailed geometrical constraints defines the explicit manipulation margin of the forbidden region for an entire articulated surgical instrument (e.g. a snake robot) rather than just the end-effector itself. Detailed experimental validation is conducted to demonstrate the speed and accuracy of the instrument navigation with and without the use of the proposed dynamic constraints. 


\section{Method}

\subsection{Construction of Dynamic Active Constraints}

Dynamic active constraints are pre-determined constraining pathways that adjust to tissue deformation in real time. This method helps the surgeon by constraining the motion within a safety margin for a prescribed path rather than discrete points. First, an obstacle-free centerline is generated as a collection of reference control points that describe the convoluted path. By using a 3D coordinate input device, the operator can place a series of control points which determine a space curve. Because of manual point placement, a refined adjustment and increment of points is conducted automatically by checking collision with the anatomical model.

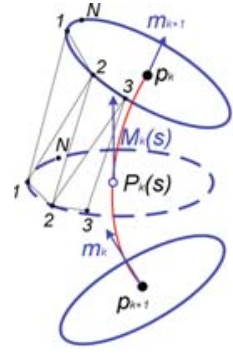

(a)

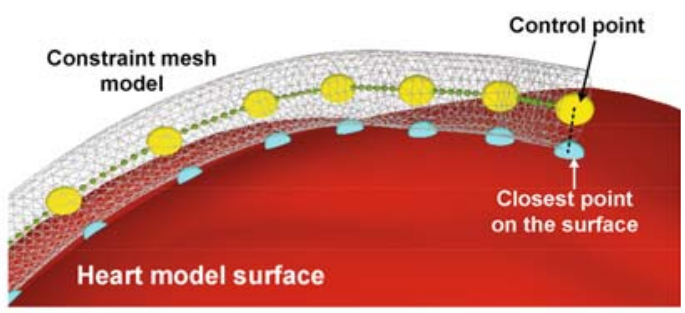

(b)

Fig. 1. (a) Triangular mesh formed between two adjacent rings of the model for active constraints; (b) the constraint represented as a tube-pathway mesh boundary along the parametric centerline

Each control point can be expanded spherically in 3D space, i.e., a margin is defined by the control radius of a sphere; considering the dimension of the adopted articulated instrument. If the distance to the epicardial surface is larger or smaller than the margin, the locations of the control points are readjusted towards the centre of the sphere. When the instrument manipulation is to follow a path within a prescribed boundary, a parametric centerline is defined as $C(s): s \in[0,1] \rightarrow \mathfrak{N}^{3}$. Hermite curve (cspline) is used in this study to represent the centerline determined by $N_{c}$ control points. The advantage of using cspline over other types of approximation is that it provides intuitive geometric manipulation for adjusting a set of parameters by which the generated curve can be adapted to the dynamic environment. For interpolation with the control points, the polynomial curve segment $C_{k}$ at $s \in(0,1)$ is defined as

$$
C_{k}(s)=h_{0,0}(s) p_{k}+h_{1,0}(s) m_{k}+h_{0,1}(s) p_{k+1}+h_{1,1}(s) m_{k+1}
$$

where $k=1, \ldots, N_{c}-1, h_{0,0}, h_{1,0}, h_{0,1}$ and $h_{1,1}$ are 3rd-order polynomial functions with respect to $s . p_{k}$ and $p_{k+1}$ are the start and end points of the curve and $m_{k}$ and $m_{k+1}$ are the corresponding tangential values at these points, where $m_{1}=p_{2}-p_{1}, m_{N c}=p_{N c}-p_{N c-1}$ and $m_{k}=\left(p_{k+1}-p_{k-1}\right) / 2$, for $k \neq 1$ and $k \neq N_{c}$. Tangent $M_{k}(s)$ at intermediate point $C_{k}(s)$ is 
interpolated as $M_{k}(s)=(1-s) m_{k}+s\left(m_{k-1}\right)$. Fig. 1(a) illustrates the rings centered at $C_{k}(s)$ and their orientations conformance to $M_{k}(s)$. Each ring is represented by a polygon of radius $r$. $N$ number of polygon vertices are aligned in an anti-clockwise direction. Before being transformed along the curve, the polygon vertices are positioned on the $X-Y$ plane and centered at the origin. The coordinates of the vertices, $X_{i}$, are determined by Eq. (2). To align the $Z$-axis of the polygonal coordinate frame parallel to $M_{k}(s)$, the equivalent rotation matrix $R(\kappa, \theta)$ has to be computed, which denotes the rotation about a unit vector $\kappa$ by an angle of $\theta$ calculated in Eq. (3). Therefore, the Cartesian coordinates of polygon vertex $X_{k i}^{\prime}$ relative to the global coordination frame can then be obtained in Eq. (4), such that the vertices are centered at point $C_{k}(s)$ and along the curve segment $k$.

$$
\begin{gathered}
X_{i}=r[\sin (2 \pi i / N), \cos (2 \pi i / N), 0]^{T} \\
\kappa_{k}(s)=\operatorname{norm}\left([0,0,1]^{T} \times M_{k}(s)\right) \text { and } \theta_{k}(s)=\cos ^{-1}\left(M_{k}(s) \cdot[0,0,1]^{T} /\left|M_{k}(s)\right|\right) \\
X_{k i}^{\prime}(s)=R\left(\kappa_{k}(s), \theta_{k}(s)\right) X_{i}+C_{k}(s)
\end{gathered}
$$

The algorithm for mesh generation interconnects the vertices between two adjacent rings, thus forming a triangular mesh structure. To provide a smooth boundary, collision checking between two adjacent rings is performed. If the two adjacent rings are found to be colliding with each other, the interconnection will be formed by choosing another ring with larger separation. As shown in Fig. 1(b), a smooth cylindrical pathway describing the active constraint is constructed. Since only point-to-object collision checking is involved, the adjustment is fast and can be conducted in real time. The objective of active constraints is to confine the instrument manipulation with the margins of the forbidden region being extended cylindrically along the centerline. The cylindrical radius is a variable that can be adjusted to allow for movement of the robot within the tunnel. It is determined by the dimension of the flexible robot and the required volumetric tolerance. For this study, it was set to $2 \mathrm{~mm}$ for all experiments. Furthermore, the upper constraints can extend beyond the pericardium to accommodate stretching.

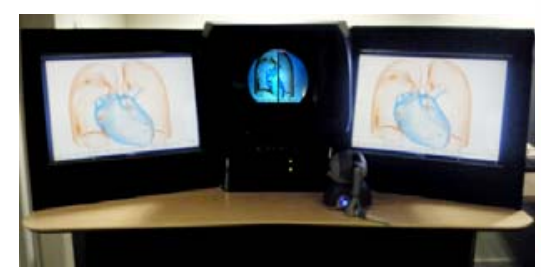

(a)

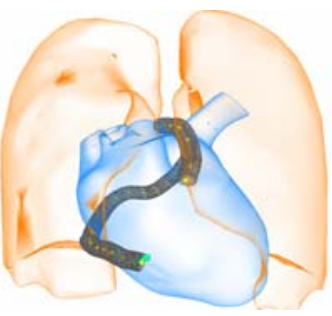

(b)

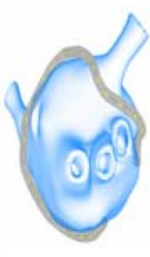

(c)

Fig. 2. The 3D auto-stereoscopic environment used for surgical navigation: (a) front view; The dynamic active constraints generated on heart model: (b) shown with the lung model and (c) viewed from top 


\subsection{Experimental Setup}

A high resolution 3D auto-stereoscopic display system (IRIS-3D, UK) was used to provide a navigation environment as shown in Fig. 2(a). The operator can view stereo images while navigating through the anatomical pathways. A synthetic simulation environment with a deforming heart and lung model was created. The 3D model was reconstructed from the CT data of a cardiac phantom (Chamberlain Group, MA, USA). The method was validated by recruiting nine subjects to assess the performance of the dynamic active constraints. Subjects were asked to operate a phantom tool device (Omni Phantom, SensAble Tech. Inc., USA) and trace a desired path on the beating heart model. To simulate ablation pathways similar to a maze procedure, dynamic active constraints were constructed and wrapped around the heart as shown in Fig. 2. To simulate realistic cardiac motion, interpolation was used between prescribed key frames including static heart reference $H$, deforming long $L$ and short $S$ cardiac axes. These were superimposed as described by Eq. (5).

$$
V(t)=V_{H}+\left(V_{S}-V_{H}\right) \frac{\sin \left(2 \pi f_{S} t\right)+1}{2}+\left(V_{L}-V_{H}\right) \frac{\sin \left(2 \pi f_{L} t\right)+1}{2}
$$

where $V_{H}, V_{L}$ and $V_{S}$ denote the vertices location at the key frames. In this experiment, the two modes of deformation were set to repeat at a rate of $f_{S} \mathrm{~Hz}$ and $f_{L} \mathrm{~Hz}$, with $f_{S}=2 f_{L}$ (e.g. $f_{S}=1,60 \mathrm{bpm}$ (beats per minute) ) in order to provide realistic periodic motion. In all experiments, $60 \mathrm{bpm}$ was chosen as the reference heart rate as the average heart rate of a healthy adult at rest is around 60 to $80 \mathrm{bpm}$. Subjects were required to operate the surgical tool tracing a desired ablation path on the heart model with $(60 \mathrm{bpm})$ and without cardiac motion. Each subject performed the task twice by using free-hand manipulation and constrained by forces generated by the phantom device. The order of the permutation was randomized to prevent bias due to learning effects. Six performance indices were recorded. They include task completion time, the maximum and average depth of collision onto the heart model, the number of collisions recorded, the total distance travelled during collision (collision path length) and the average of the total distance when the tool deviated from the desired trajectory (path deviation). Data analysis was performed by using a between-group comparison statistical test (One-Way ANOVA with Bonferroni test, SPSS Inc., Chicago, IL). Four groups were compared: 1) Static heart without constraints; 2) Static heart with constraints; 3) Heart beating at $60 \mathrm{bpm}$ without constraints; 4) Heart beating at $60 \mathrm{bpm}$ with constraints. Each of them is mutually independent among the six performance indices. The main purpose of the performance metrics is to demonstrate the confidence gain by the use of dynamic active constraints. These metrics are related to the deviation of the instrument from the ideal path. Instrumenttissue forces were not rendered in the case of free-hand operation. In order to assess how the heart rate affects the accuracy of manipulation, the experiment as described above was also conducted with the heart beating rate varying from 60 to $120 \mathrm{bpm}$.

\section{Results}

Using multiple comparison tests, statistically significant differences $(\alpha<0.05)$ occurred between the four groups among all six performance indices. More specifically, 
obtained $\alpha$-values ranged within [0, 0.008]. The mean and standard deviation of the six performance indices for all subjects, with and without the use of constraints, are summarized in Table 1. It is evident that with the introduction of dynamic active constraints, the overall performance has been improved significantly. For both static and dynamic experiments, improved scores are obtained for all indices when active constraints are activated. No collision was found when subjects traced a desired path on the static heart model with constraints. Overall, navigation with constraints has shown reduced task completion time and path deviation. Furthermore, this performance gain seems to be independent of the static or dynamic environment, showing less than $1 \%$ variance. Fig. 3(a) illustrates the path deviation for one of the subjects studied. It can be seen that path deviation with constraints is less than that without constraints, maintained at a steady level of $2 \mathrm{~mm}$. This was attributed to the fact that the subject followed the inner surface of the pathway during operation. With the use of constraints, the reduced path variation reflects the confidence of the operator during $3 \mathrm{D}$ maneuver. Fig. 3(b) shows that the path-to-path variance of path deviation for the entire experiment was less than $1 \mathrm{~mm}$. Unsurprisingly, the trajectory of the tool tip while performing path following with constraints is close to the desired path as shown in Fig. $3(\mathrm{c})$. These experiments were repeated with a range of heart rates (60 to $120 \mathrm{bpm})$ and the corresponding results are shown in Table 2, where the values shown are normalized performance gains for each index with the use of constraints.

Standardization of data was achieved at different frequencies from a single subject. In Table 2, the percentage change was calculated by using the performance indices of free-hand operation (i.e., without the use of active constraints) as the reference. It is evident from these results that the overall performance gain is maintained as the heart rate increases. During high-frequency motion, the overall hand-eye coordination is generally deteriorated, highlighting the need of virtual motion stabilization in these cases. To better visualize the change in the margins associated with these gains, Fig. 4

Table 1. Summary of changes in performance indices with and without the use of dynamic active constraints (averaged across the nine subjects studied)

\begin{tabular}{llllccccc}
\hline & \multicolumn{3}{c}{ Without constraints } & \multicolumn{3}{c}{ With constraints } \\
\cline { 2 - 9 } & \multicolumn{2}{c}{$\begin{array}{c}\text { Static } \\
\text { heart }\end{array}$} & $\begin{array}{c}\text { Beating heart } \\
(60 \mathrm{bpm})\end{array}$ & $\begin{array}{c}\text { Static } \\
\text { heart }\end{array}$ & $\begin{array}{c}\text { Beating heart } \\
(60 \text { bpm })\end{array}$ \\
\hline & mean & SD & mean & SD & mean & SD & mean & SD \\
\hline Completion time (sec) & 35.9 & $(15.3)$ & 37.6 & $(16.6)$ & 12.9 & $(10.1)$ & 11.0 & $(5.1)$ \\
Max collision depth, mm & 14.6 & $(8.5)$ & 15.0 & $(11.8)$ & 0.0 & $(0.0)$ & 0.7 & $(0.9)$ \\
Mean collision depth, mm & 7.5 & $(4.8)$ & 6.8 & $(5.6)$ & 0.0 & $(0.0)$ & 0.7 & $(0.8)$ \\
No. of collisions & 5.0 & $(2.4)$ & 7.0 & $(2.6)$ & 0.0 & $(0.0)$ & 0.7 & $(0.9)$ \\
Collision path length, mm & 123.8 & $(59.7)$ & 112.0 & $(90.9)$ & 0.0 & $(0.0)$ & 2.9 & $(3.9)$ \\
Path deviation, mm & 8.4 & $(4.6)$ & 12.8 & $(9.4)$ & 2.0 & $(0.0)$ & 1.7 & $(0.3)$ \\
\hline
\end{tabular}

Table 2. Percentage change in performance indices for varying heart rate (60-120 bpm)

\begin{tabular}{lccccccc}
\hline Heart rate (bpm) & 60 & 70 & 80 & 90 & 100 & 110 & 120 \\
\hline Completion time (\%) & 60.1 & 60.3 & 59.0 & 45.3 & 66.7 & 76.8 & 50.1 \\
Path deviation (\%) & 23.9 & 84.6 & 42.9 & 34.0 & 58.3 & 63.8 & 31.0 \\
\hline
\end{tabular}




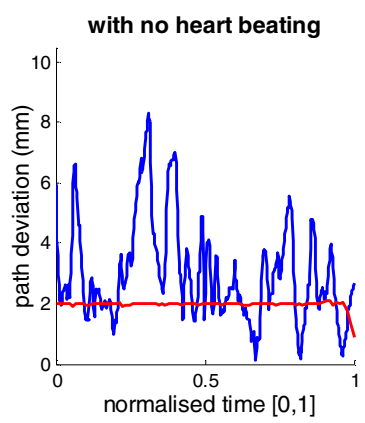

(a)

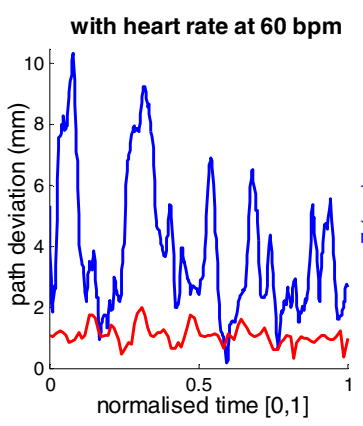

(b)

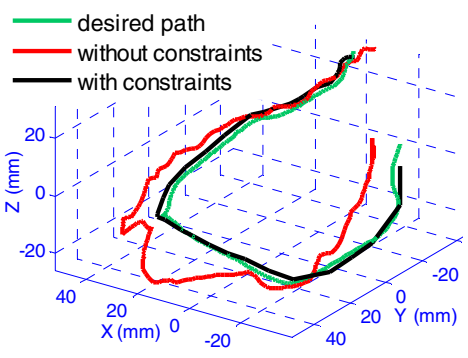

(c)

Fig. 3. Example path deviation plots for one of the subjects studied, showing the absolute path deviation and variance throughout the procedure with and without constraints when the heart is static (a) and beating (b). (c) Trajectory of the virtual tool tip in following the desired path.

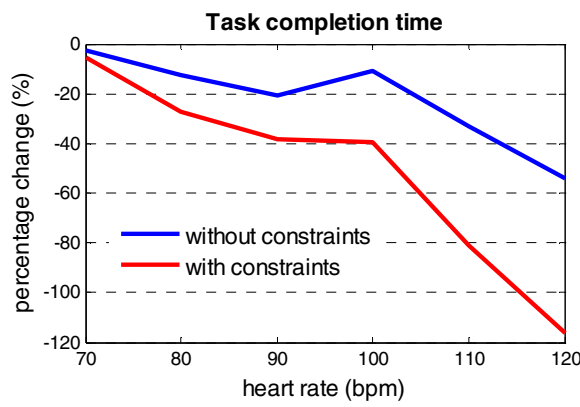

(a)

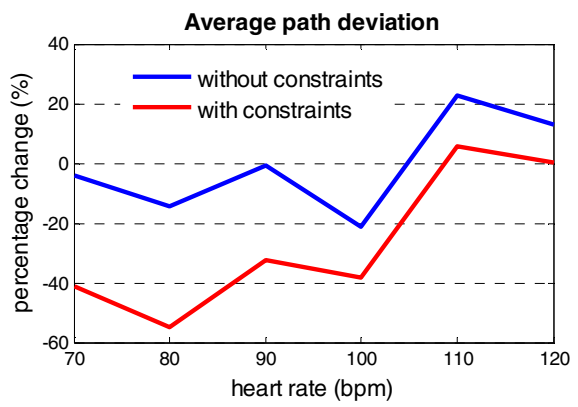

(b)

Fig. 4. Percentage change in task completion time (a) and average path deviation (b) with and without the use of constraints normalized against the respective indices at $60 \mathrm{bpm}$

illustrates the trend of the task completion time and average path deviation with increasing heart rate. In this figure, the percentage change is calculated by using the respective performance indices at $60 \mathrm{bpm}$ as the reference. In Fig. 4(a), a decrease (more negative) in task completion time implies that shorter time is required to complete the task for both cases. Similarly, increased path-following errors are indicated by the percentage increase of path deviation as shown in Fig. 4(b). This can be attributed to the fact that motor tracking is worse while the desired path is moving faster at higher heart rates. As a result, the subject was not able to perform detailed tracking of a particular region.

\section{Discussion and Conclusions}

In this paper, a real-time modeling scheme for dynamic active constraints is proposed. Validation through the use of a simulated beating heart model demonstrates its ability in adapting to changes of tissue morphology. Experimental validation has shown 
statistically significant improvement in terms of speed, accuracy and minimized tissue damage when dynamic active constraints are introduced. The clinical relevance of the study is that in many cardiac intervention procedures, particularly the maze procedure, it is not appropriate to use the pericardium and the heart itself as a physical constraint to limit the motion of the robotic probe normal to the epicardial surface. Forces normal to the epicardial surface can penetrate the myocardium injuring coronary vessels or perforating the pericardium and damaging sensitive nerves such as the phrenic nerve. In this case, flexible, non-articulated devices may not provide accurate 3D navigation. This has motivated the development of hyper-redundant flexible robots with 3D dynamic active constraints. The proposed motion constraints concept can readily incorporate manipulation margin of long articulated surgical instruments rather than just the end-effectors. Such a scheme is also suitable for integrating other navigation schemes, e.g., gaze contingent motor channeling [10], for further enhancing the surgeon's hand-eye coordination.

Acknowledgments. The work described in this paper was supported by grants from the UK EPSRC (DT/E011101/1, EP/D057213/1), TSB and the Welcome Trust.

\section{References}

1. Hornero, F., Rodríguez, I., Bueno, M., Buendía, J., Dalmau, M.J., Canovas, S., Gil, O., Garcia, R., Montero, J.A.: Surgical ablation of permanent atrial fibrillation by means of maze radiofrequency: mid-term results. J. Card. Surg. 19, 383-388 (2004)

2. Raman, J.S., Seevanayagam, S., Storer, M., Power, J.M.: Combined endocardial and epicardial radiofrequency ablation of right and left atria in the treatment of atrial fibrillation. Ann. Thorac. Surg. 72, 1096-1099 (2001)

3. Rosenberg, L.B.: Virtual fixtures: Perceptual tools for telerobotic manipulation. In: Proc. of the IEEE Annual International Symposium on Virtual Reality, pp. 76-82 (1993)

4. Okamura, A.M.: Methods for haptic feedback in teleoperated robot-assisted surgery. Industrial Robot: An International Journal 31, 499-508 (2004)

5. Davies, B., Jakopec, M., Harris, S.J., Baena, F.R.Y., Barrett, A., Evangelidis, A., Gomes, P., Henckel, J., Cobb, J.: Active-constraint robotics for surgery. Proc. of the IEEE 94, 1696-1704 (2006)

6. Li, M., Ishii, M., Taylor, R.H.: Spatial motion constraints using virtual fixtures generated by anatomy. IEEE Trans. Robotics 23, 4-19 (2007)

7. Ren, J., Patel, R.V., McIsaac, K.A., Guiraudon, G., Peters, T.M.: Dynamic 3-D virtual fixtures for minimally invasive beating heart procedures. IEEE Trans. on Medical Imaging 27, 1061-1070 (2008)

8. Zenati, M.A., Bonanomi, G., Chin, A.K., Schwartzman, D.: Left heart pacing lead implantation using subxiphoid videopericardioscopy. J. Cardiovasc Electrophysiol. 14, 949-953 (2003)

9. Zenati, M.A., Shalaby, A., Eisenman, G., Nosbisch, J., McGarvey, J., Ota, T.: Epicardial left ventricular mapping using subxiphoid video pericardioscopy. Ann. Thorac. Surg. 84, 2106-2107 (2007)

10. Mylonas, G.P., Kwok, K.W., Darzi, A., Yang, G.-Z.: Gaze-contingent motor channelling and haptic constraints for minimally invasive robotic surgery. In: Proc. of Int. Conf. on Medical Image Computing and Computer-Assisted Intervention, vol. 11, pp. 676-683 (2008) 